\title{
Aleukemic Leukemia Cutis Manifesting with Disseminated Nodular Eruptions and a Plaque Preceding Acute Monocytic Leukemia: A Case Report
}

\author{
Ipek Yonal $^{\mathrm{a}}$ Fehmi Hindilerden $^{\mathrm{c}}$ Raif Coskun $^{\mathrm{a}}$ \\ Oner Ibrahim Dogan ${ }^{b}$ Meliha Nalcaci ${ }^{a}$ \\ ${ }^{a}$ Division of Hematology, Department of Internal Medicine, and ${ }^{b}$ Department of \\ Pathology, Medical Faculty, Istanbul University, and 'Division of Hematology, \\ Department of Internal Medicine, Istanbul Bilim University, Istanbul, Turkey
}

\section{Key Words}

Leukemia cutis - Acute monocytic leukemia - Aleukemic leukemia cutis - Atypical monocytoid cells $\cdot$ Disseminated nodular eruptions

\begin{abstract}
Aleukemic leukemia cutis (ALC), a discrete tumor of leukemic cells involving the skin, may be the first manifestation of acute myeloid leukemia, preceding the onset in marrow and blood by months and years. ALC is often difficult to diagnose and is associated with a dismal prognosis. A 63-year-old male presented with nodular swellings on the face, a plaque extending over the right shoulder and multiple enlarged cervical lymph nodes. The skin biopsy of the plaque lesion showed a diffuse neoplastic infiltration extending from the dermis to subcutaneous tissue with diffuse positivity for myeloperoxidase and focal positivity for CD34 on immunohistochemical staining. The diagnosis was leukemia cutis. One month later, acute monocytic leukemia (FAB AML-M5b) was diagnosed. The patient died on the seventh month of diagnosis.
\end{abstract}

\section{Introduction}

Leukemia cutis (LC) denotes the invasion of neoplastic leukocytes or their precursors into the epidermis, dermis, or subcutis, presenting with various cutaneous lesions including papules, macules, plaques, nodules, ecchymoses, palpable purpura, 
and ulcerative lesions [1-9]. The lesions often appear as violaceous nodules. LC is more common in the monocytic subtype of acute myeloid leukemia (AML). The lesions may be localized or disseminated, presenting at any site over the skin. In most cases of LC, systemic disease is already present; only $7 \%$ of cases are aleukemic. In $13 \%$ of cases of LC, AML is the underlying disease [10]. Aleukemic leukemia cutis (ALC) is the infiltration of skin by blasts prior to appearance in peripheral blood and bone marrow. The primary extramedullary presentation of acute leukemia is extremely rare and usually associated with early bone marrow relapse and poor therapy outcome [11]. The early recognition of the lesions of LC should be emphasized.

\section{Case}

A 63-year-old man was admitted to our hematology department with extensive nodular lesions on the trunk, submental area, forehead, scalp and a plaque extending over the right shoulder. Four months before admission to our department, he had been examined for swellings on the left side of his face and the right shoulder. On physical examination, a firm, $4-\mathrm{cm}$ mass inferior to the left zygomatic arch, a 15-cm brown pigmented, indurated mass extending from the lateral side of sternocleidomastoid region to the deltoid muscle and multiple submandibular lympadenopathies measuring a maximum diameter of $3.5 \mathrm{~cm}$ were noted. At that time, his laboratory parameters were normal, including a complete blood count and blood chemistries. MRI scan of the face revealed a $4.2 \times$ $2.3 \times 3.8-\mathrm{cm}$ slightly lobulated mass at the inferior of the left zygomatic arch, extending from the nasolabial sulcus to the mandibular corpus and the lateral maxilla. Also, multiple lymphadenopathies with a maximal diameter of $3.4 \mathrm{~cm}$ were present at the submandibular, jugulodigastric regions and the posterior spinal accessory chain. A $15 \times 10$-cm mass on the right shoulder joint extending from the lateral side of sternocleidomastoid region to the deltoid muscle was evident on MRI. A skin biopsy had been obtained from the plaque lesion on the right shoulder. One month after the skin biopsy, the patient was evaluated for worsening malaise and fatigue. The laboratory results were as follows: leukocyte $46,000 / \mathrm{mm}^{3}$ (monocyte $44 \%$, neutrophil 39\%), hemoglobin $9 \mathrm{~g} / \mathrm{dl}$, platelet $64,000 / \mathrm{mm}^{3}$, LDH 1,109 IU/l (normal range, 240-480 IU/l). The skin biopsy of the plaque lesion showed diffuse neoplastic infiltration with positivity for myeloperoxidase (MPO) and CD34 on immunohistochemical staining. On peripheral blood smear, diffuse myeloblastic infiltration was evident (fig. 1). The bone marrow aspirate was hypercellular, with $22 \%$ monocytoid blasts. Immunophenotypic analysis of peripheral blood was positive for CD13, CD15, CD25, CD33, CD34, CD45, HLA-DR and MPO. These findings were compatible with acute monocytic leukemia (FAB AML-M5b). Having presented with skin involvement one month prior to the onset of AML-M5b, the final diagnosis was ALC. The remission induction regimen consisted of cytarabine $100 \mathrm{mg} / \mathrm{m}^{2}$ daily on days $1-7$ and daunorubicin $60 \mathrm{mg} / \mathrm{m}^{2}$ daily on days 1-3. On the 2nd week of chemotherapy, febrile neutropenia developed. Imipenem-susceptible Pseudomonas aeruginosa was grown on culture media. After seven days of persistent fever under imipenem, empirical antifungal treatment with amphotericin-B was added and was subsequently substituted by voricanozole four days later due to ongoing fever. On day 28, there were no myeloblasts on peripheral blood smear and bone marrow examination, but there was only $50 \%$ regression of skin lesions. Therefore, the same chemotherapy regimen was administered for reinduction. On day 28 of reinduction, physical examination revealed hepatosplenomegaly, extensive brown pigmented nodular lesions on the trunk, submental area, forehead, scalp and a plaque extending over the right shoulder. Then, the patient was referred to our hematology department. The reexamination of the previous skin biopsy specimen revealed diffuse neoplastic infiltration extending from the dermis to subcutaneous tissue with diffuse positivity for MPO and focal positivity for CD34 on immunohistochemical staining, which were findings compatible with the diagnosis of LC (fig. 2 ). On his physical examination at our clinic, progressive skin infiltrations as nodular eruptions on scalp, forehead and trunk and a plaque on the right shoulder were noted (fig. $3 \mathrm{a}-\mathrm{c}$ ). At the time of admission, the laboratory results were as follows: leukocyte $2,000 / \mathrm{mm}^{3}$ (monocyte $42 \%$, neutrophil $32 \%$ ), hemoglobin $8 \mathrm{~g} / \mathrm{dl}$, platelet 76,000/ $\mathrm{mm}^{3}$, serum LDH 1,335 IU/l and ESR $96 \mathrm{~mm} / \mathrm{h}$. The bone marrow aspirate showed $20 \%$ blastic infiltration. Histopathologic examination disclosed a hypercellular marrow with a medium to diffuse increase in reticulin fibers and $20 \%$ immature cells staining positive for MPO, CD33, CD34. Diagnosed as refractory disease, FLAG chemotherapy, a more aggressive regimen consisting of granulocyte colony-stimulating factor, a 5-day course of 30 
$\mathrm{mg} / \mathrm{m}^{2} /$ day fludarabine and $2 \mathrm{~g} / \mathrm{m}^{2} /$ day cytosine arabinoside was initiated. On the 8 th day of chemotherapy, neutropenia developed. Subsequently, urinary tract infection caused by enterococci was diagnosed and treated with piperacillin/tazobactam for 14 days. On the 10th day of induction chemotherapy, the nodular lesion on the face almost completely regressed, the pigmentation of nodular lesions on the trunk began to fade, and the indurated plaque on the right shoulder healed with hyperpigmentation (fig. 3d-f). On the 23rd day of chemotherapy, the patient developed neutropenic fever. Escherichia coli was isolated on blood culture and imipenem was started. Later, ampiric antifungal treatment with amphotericin-B was added because of ongoing fever. However, amphotericin-B was substituted for caspofungin for allergic reaction. On the 28th day of chemotherapy, LDH returned to normal and the skin infiltrates largely disappeared. However, on the 45th day of chemotherapy, new disseminated nodular eruptions reappeared on the forehead, face and trunk. In addition, a 5-cm firm, endurated nodular lesion at the left submental area, a 1-cm mass at the left nasal cavity and a $15 \times 10-\mathrm{cm}$ mass on the right shoulder joint extending from the lateral side of sternocleidomastoid region to the deltoid muscle were detected. There was considerable venous distention of the right arm due to the occlusion of the right subclavian vein confirmed by venous Doppler USG (fig. 4). The blood count was as follows: leukocyte $4,730 / \mathrm{mm}^{3}$ (monocyte $73 \%$, neutrophil 17\%), hemoglobin $7 \mathrm{~g} / \mathrm{dl}$, and platelet $24,000 / \mathrm{mm}^{3}$. Peripheral blood smear revealed $92 \%$ myeloblasts. Serum LDH was 1,466 IU/l. Emergent chemotherapy to relieve pressure symptoms was initiated with cytarabine $100 \mathrm{mg} / \mathrm{m}^{2}$ for 7 days and idarubicin $12 \mathrm{mg} / \mathrm{m}^{2}$ for 3 days. On the 7 th day of chemotherapy, febrile neutropenia was diagnosed. The blood culture was positive for Pseudomonas aeruginosa. Meropenem and ciprofloxacin were started. After 14 days, trimethoprim-sulphonamide was added for ongoing fever. On the 28th day of chemotherapy, all the skin lesions except for those on the trunk showed significant clinical improvement. The distention of the right arm disappeared. The patient had prolonged neutropenia for 3 months. The patient succumbed to his disease 6 months after initial diagnosis.

\section{Discussion}

Skin involvement in AML may present as nonspecific lesions, LC, or granulocytic (myeloid) sarcoma of skin and subcutis [12-17]. Nonspecific skin lesions encountered in AML are macules, papules, vesicles, pyoderma gangrenosum, vasculitis, neutrophilic dermatitis (Sweet syndrome), cutis vertices gyrata, and erythema multiforme or nodosum $[12,13]$. Present in up to $13 \%$ of AML patients, LC is mostly associated with monocytic or myelomonocytic subtypes [18]. The review by Desch and Smoller [19] of 123 skin biopsies obtained from leukemic patients showed LC to be present in 37 (30\%) patients, 23 of whom had AML or myelodysplastic syndrome. Of note, however, was the presence of a cutaneous process other than LC, mainly infection or drug reaction in the skin biopsies of AML patients. The large series by Boggs et al. [20] demonstrated LC to be present in 11.0 and $1.3 \%$ of patients with AML and acute lymphoblastic leukemia, respectively. Acute monocytic leukemia (FAB-M5) is disproportionately represented [20]. LC usually presents either concomitantly or after the diagnosis of systemic leukemia [18]. Skin involvement preceding marrow and blood involvement is rare [17].

ALC defines a rare form of leukemia in which the invasion of leukemic blasts into the skin occurs at least one month prior to the appearance of disease in peripheral blood or bone marrow [21]. Cases of ALC reported thus far show little or no harmony in their clinical presentation. ALC clinically presents with multiple papules, nodules, or infiltrated plaques with a red-brown or plum-colored surface [22]. A literature review showed that ALC was commonly misdiagnosed [23]. Its recognition is important because early diagnosis may enable a better prognosis. 
ALC presents at least one month before peripheral blood or bone marrow involvement. When our case presented with nodular swellings on the face, a plaque extending over the right shoulder, all laboratory findings were normal. Yet, a month later, he was diagnosed with acute myeloid leukemia.

In a case series of $31 \mathrm{ALC}$ patients by Chang et al. [24], the most common sites of extramedullary involvement other than the skin were the lymph nodes (8 patients) followed by the spleen ( 2 patients). In addition to the disseminated nodular eruptions and the plaque lesion, our case presented with multiple enlarged cervical lymph nodes. Also, two months after the remission-induction therapy, his physical examination revealed splenomegaly.

The diagnosis of LC requires a skin biopsy. Dense infiltrate comprising a linear array of pleomorphic leukemic cells in the reticular dermis with routine hematoxylin-eosin (H\&E) staining are suggestive histological features. ALC must be distinguished by immunohistochemical studies from lymphoproliferative disorders. In our case, immunohistochemical study demonstrated positivity for CD34 and MPO, confirming the diagnosis of LC.

In ALC, early marrow relapse and poor treatment response is the usual outcome. The mean survival time is from 3 to 30 months once leukemic cells appear in peripheral blood or bone marrow $[22,24]$. Our patient had refractory disease and died 6 months after initial diagnosis.

Patterns of skin involvement in ALC are particularly variable requiring a high degree of clinical suspicion. Unusual skin eruptions with nodules and plaques may be the presenting feature of ALC, as in our case. Early recognition of LC in aleukemic patients and prompt hematological treatment is key to survival. Immunohistochemistry is necessary to confirm the diagnosis of ALC. Because of the rarity of the disease and the lack of controlled studies, there is no consensus on the treatment of choice for ALC. Radiotherapy, chemotherapy, and total body electron therapy have yielded variable results $[6,22,24-28]$.

\section{Disclosure Statement}

The authors have no conflicts of interests to declare. 


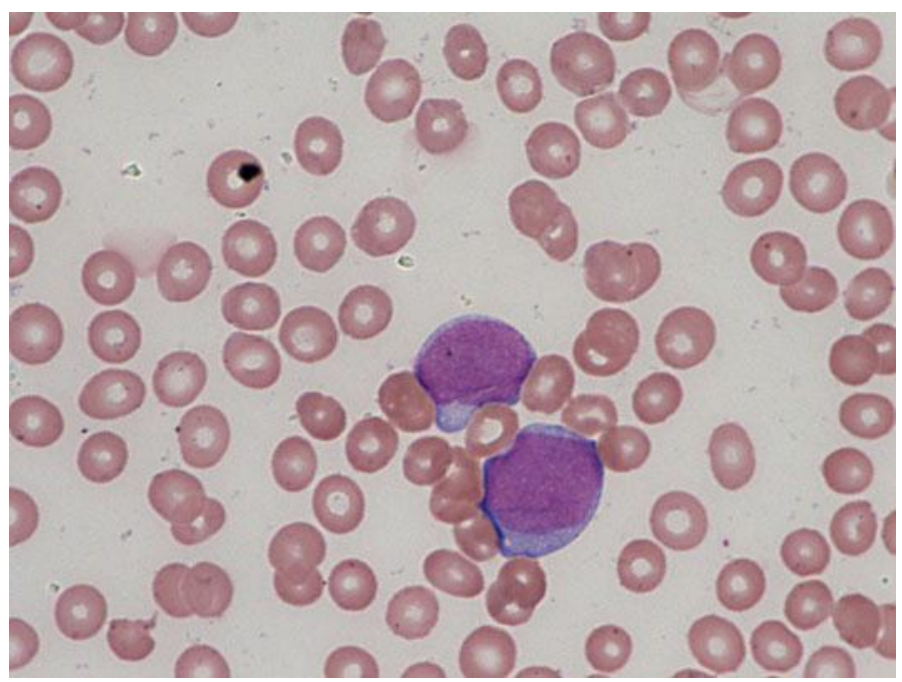

Fig. 1. Peripheral smear with atypical monocytoid cells (FAB AML-M5b).

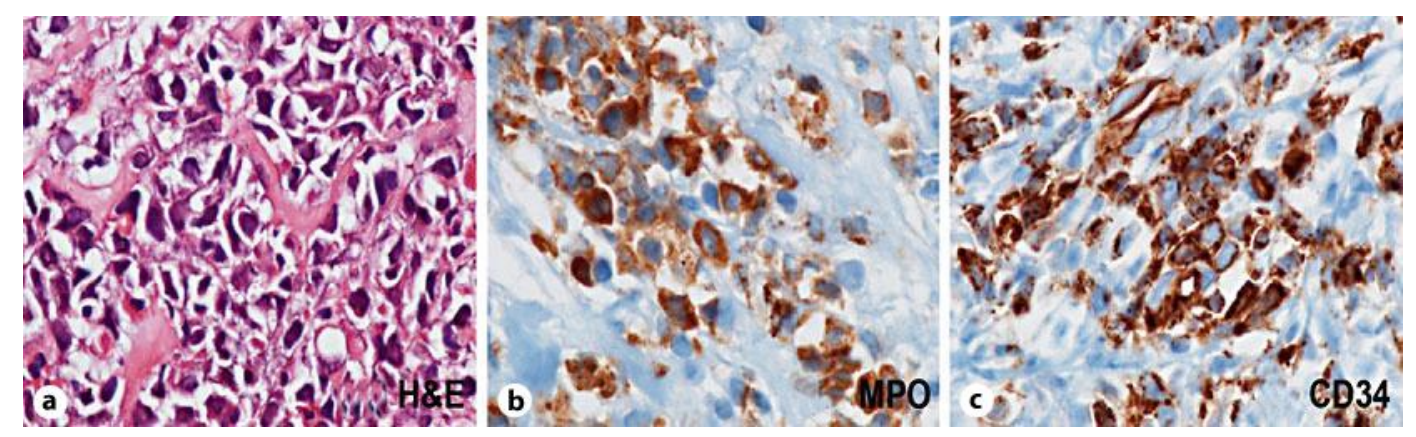

Fig. 2. a The skin biopsy specimen of the plaque lesion showed diffuse neoplastic infiltration in the dermis and subcutis $(\mathrm{H} \& \mathrm{E}, \times 400)$. Immunohistochemical features. The infiltrated cells were diffuse positive for MPO $(\mathbf{b} ; \times 400)$ and focally positive for CD34 (c; $\times 400)$. 

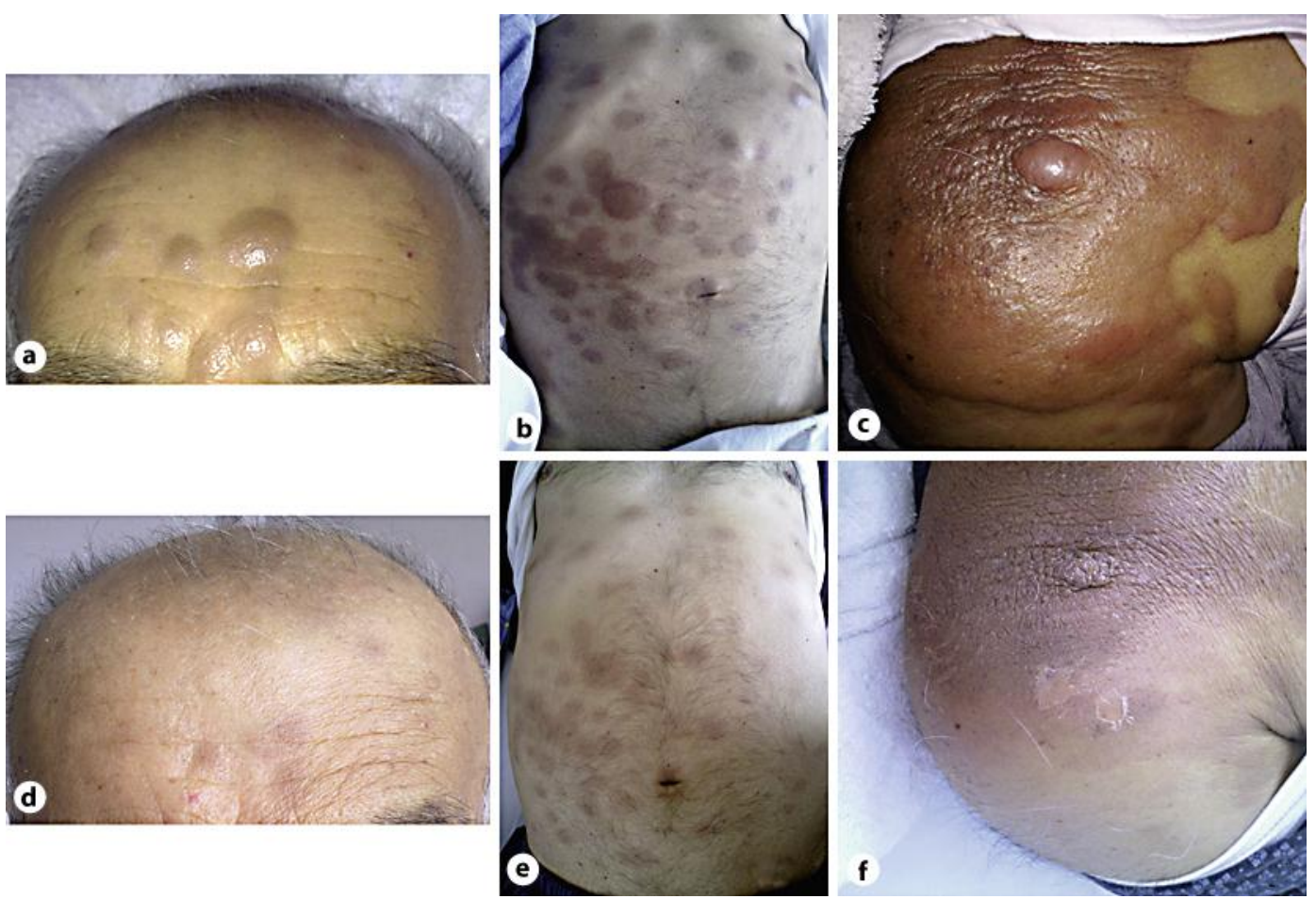

Fig. 3. a-c The appearance of the lesions at admission to our center after two courses of remission induction with cytarabine and daunorubicin. Nodular eruptions on the forehead (a), trunk (b) and a plaque lesion extending over the right shoulder $(\mathbf{c})$. $\mathbf{d}-\mathbf{f}$ The healing lesions on the 10th day of FLAG induction chemotherapy. The appearance of the forehead (d), trunk (e) and right shoulder (f). 


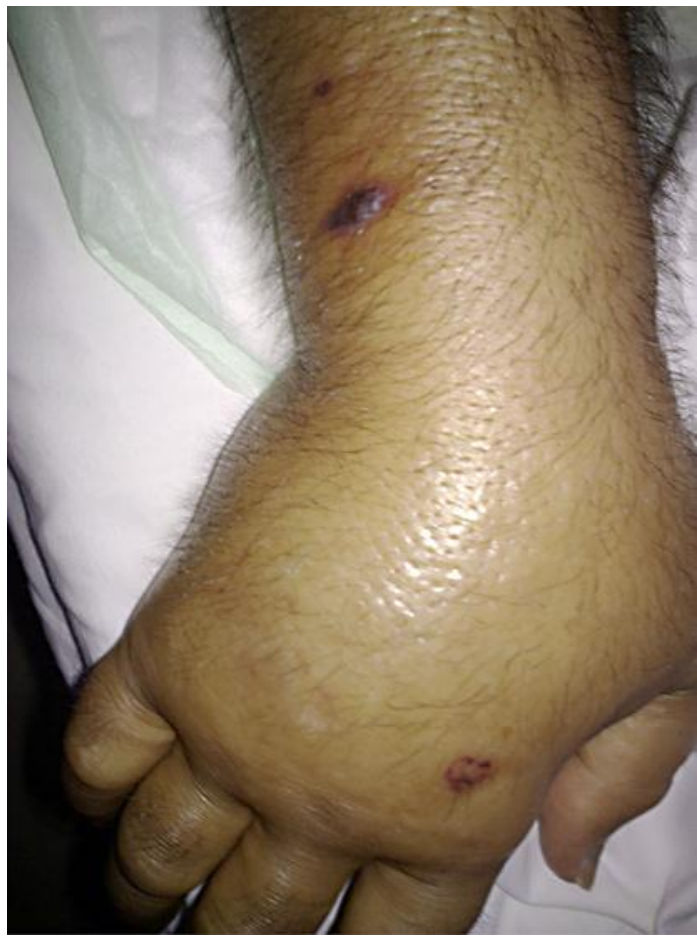

Fig. 4. The distended right forearm due to the occlusion of the right subclavian vein by the mass on the right shoulder.

\section{References}

1 Husak R, Blume-Peytaki U, Orfanos C: Aleukemic leukemia cutis in an adolescent boy. N Engl J Med 1999;340:893-894.

-2 Chang HY, Wong KM, Bosenberg M, et al: Myelogenous leukemia cutis resembling stasis dermatitis. J Am Acad Dermatol 2003;49:128-129.

-3 Hahn WC, Jones D, Leavitt P, et al: Leukemia cutis. Diagnosis in oncology. J Clin Oncol 1997;15:21702171.

4 Su WP, Buechner SA, Li CY: Clinicopathologic correlations in leukemia cutis. J Am Acad Dermatol 1984;11:121-128.

5 Baer MR, Barcos M, Farrel H, et al: Acute myelogenous leukemia with leukemia cutis. Cancer 1989;63:2192-2200.

6 Ohno S, Yokoo T, Ohta M, et al: Aleukemic leukemia cutis. J Am Acad Dermatol 1990;22:374-377.

7 Longacre TA, Smoller BR: Leukemia cutis. Analysis of 50 biopsy-proven cases with an emphasis on occurrence in myelodysplastic syndromes. Am J Clin Pathol 1993;100:276-284.

$>8$ Bourantas K, Malamou-Mitsi VD, Christou L, et al: Cutaneous vasculitis as the initial manifestation in acute myelomonocytic leukemia. Ann Intern Med 1994;121:942-944.

-9 Sepp N, Radaszkiewicz T, Meijer CJL, et al: Specific skin manifestations in acute leukemia with monocytic differentiation. A morphologic and immunohistochemical study of 11 cases. Cancer 1993;71:124-130.

10 Rencic A, Ramnarayanan J: Leukemia cutis. eMedicine Dermatology [last updated on Jun 7th, 2005]. Available from: http://www.emedicine.com/derm/topic924.htm [last accessed on Aug 24th, 2006].

11 Barzilai A, Lyakhovitsky A, Goldberg I, et al: Aleukemic monocytic leukemia cutis. Cutis 2002;69:301304.

12 Kaiserling E, Horny H-P, Geerts M-L, Schmid U: Skin involvement in myelogenous leukemia. Morphologic and immunophenotypic heterogeneity of skin infiltrates. Mod Pathol 1994;7:771. 
13 Longacre TA, Smoller BR: Leukemia cutis. Analysis of 50 biopsy-proven cases with an emphasis on occurrences in myelodysplastic syndromes. Am J Clin Pathol 1993;100:276-284.

14 Shaikh BS, Frantz E, Lookingbill DP: Histologically proven leukemia cutis carries a poor prognosis in acute nonlymphocytic leukemia. Cutis 1987;39:57.

15 Sipp N, Radaszkiemicz T, Meijer CJLM, et al: Specific skin manifestations in acute leukemia with monocytic differentiation. Cancer 1993;71:124.

16 Baer MR, Barcos M, Farrell H, et al: Acute myelogenous leukemia in leukemia cutis. Cancer 1989;63:2192.

17 Long JC, Mihm MC: Multiple granulocytic tumors of the skin. Report of six cases of myelogenous leukemia with initial manifestations in the skin. Cancer 1977;39:2004.

18 Ratnam KV, Khor CJ, Su WP: Leukemia cutis. Dermatol Clin 1994;12:419-431.

19 Desch JK, Smoller BR: The spectrum of cutaneous disease in leukemias. J Cutan Pathol 1993;20:407-410.

20 Boggs DR, Wintrobe MM, Cartwright GE: Acute leukemias: analysis of 322 cases and review of the literature. Medicine 1962;41:163.

21 Horlick HP, Silvers DN, Knobler EH, Cole JT: Acute myelomonocytic leukemia presenting as a benignappearing cutaneous eruption. Arch Dermatol 1990;126:653-656.

22 Okun MM, Fitzgibbon J, Nahass GT, Forsman K: Aleukemic leukemia cutis, myeloid subtype. Eur J Dermatol 1995;5:290-293.

23 Wilkins R, Janes S: Aleukaemic leukaemia cutis: case report and review of the literature. Clin Lab Haematol 2004;26:73-75.

24 Chang H, Shih LY, Kuo TT: Primary aleukemic myeloid leukemia cutis treated successfully with combination chemotherapy: report of a case and review of the literature. Ann Hematol 2003;82:435439.

25 Lee B, Fatterpekar GM, Kim W, Som PM: Granulocytic sarcoma of the temporal bone. AJNR Am J Neuroradiol 2002;23:1497-1499.

26 Török L, Lueff S, Garay G, Tápai M: Monocytic aleukemic leukemia cutis. J Eur Acad Dermatol Venerol 1999;13:54-58.

27 Tomasini C, Quaglino P, Novelli M, Fierro MT: 'Aleukemic' granulomatous leukemia cutis. Am J Dermatopathol 1998;20:417-421.

-28 Imanaka K, Fujiwara K, Satoh K, Kuroda Y, Takahashi M, Sadatoh N, et al: A case of aleukemic monocytic leukemia cutis treated with total body electron therapy. Radiat Med 1988;6:229-231. 